\title{
IgG Synthesis Rate
}

National Cancer Institute

\section{Source}

National Cancer Institute. Ig G Synthesis Rate. NCI Thesaurus. Code C147375.

The determination of the IgG synthesis rate in a biological specimen. 